\title{
La elección de centro como mecanismo de segregación social
}

\author{
The choice of school as a mechanism for social segregation
}

\author{
José Luis Bernal Agudo \\ Carlos Vera Báez \\ Universidad de Zaragoza
}

Recibido: 12/09/2019

Aceptado: 27/11/2019

\begin{abstract}
The aim of this article is to provide more evidence regarding the effects of the free choice of school on equal opportunities, and, thus, on the equity of the educational system, based on the experiences and statements of families themselves. This work is part of a much broader empirical study carried out in Zaragoza in 2013-2015, entitled "Choosing a school in Aragon: parental reasoning and strategies in choosing a school"). The interviews (43 done in depth) and questionnaires (covering 1837 families) raised three factors that determine parental participation in the selection process: scope, diversity, and quality of the educational offer. Two variables are highlighted that strongly affect family participation in the choice of school: the level of parental education, and family income, which determine the fact that, although there are eligible schools, they are not always accessible to all families. All have the opportunity to choose, but those opportunities are fewer for those who have less. That direct relationship has a name: inequality, functioning as a silent but effective mechanism for social segregation. The conclusion is that freedom of choice per se does not guarantee equal opportunities, and that, if it does not go hand-in-hand with policies aimed at reducing the initial inequalities that exist between families, freedom of choice becomes a subliminal form of social segregation that exists between families, freedom of choice becomes a subliminal form of social segregation.
\end{abstract}

KEY WORDS: Social inequality; Choice of school; School segregation; Equal opportunity; Social mobility.

\section{RESUMEN}

El presente artículo pretende aportar más evidencias acerca de los efectos de la libre elección de centro escolar en la igualdad de oportunidades y, por tanto, en la equidad del sistema educativo, a partir de las experiencias y discurso de las propias familias. Este trabajo es parte de un estudio empírico mucho más amplio realizado en Zaragoza durante los años 2013-2015, titulado La elección de centro escolar en Aragón: Razonamientos y estrategias de los padres en la elección de centro escolar. De las entrevistas (43 en profundidad) y cuestionarios (1837 familias) se plantean tres factores que determinan la participación de los padres en el proceso de elección: amplitud, diversidad y calidad de la oferta educativa. Se resaltan dos variables que condicionan fuertemente la participación de las familias en la elección de centro: el nivel de formación de los padres y la renta familiar, que determinan que, aunque haya colegios elegibles, no siempre son accesibles a todas las familias. Todos tienen oportunidades para elegir, pero estas se reducen para los que tienen menos. Esta relación positiva tiene un nombre: desigualdad, funcionando como un mecanismo silencioso pero eficaz de segregación social. Se concluye que la libertad de elección per se no garantiza una igualdad de oportunidades y que, si no va acompañada de políticas que busquen reducir las desigualdades de partida que hay entre las familias, la libertad de elección se convierte en una forma subliminal de segregación social.

PALABRAS CLAVE: Desigualdad social; Elección de la escuela; Segregación escolar; Igualdad de oportunidades; Movilidad social.

Autor de correspondencia

José Luis Bernal Agudo, Departamento de Ciencias de la Educación, Universidad de Zaragoza. Email jbernal@unizar.es. ORCID: https://orcid.org/0000-0002-3437-2406 


\section{Introducción}

El capitalismo como sistema económico-social genera desigualdad en sí mismo, siendo el Estado el responsable de redistribuir la riqueza y compensar las desigualdades a través del denominado Estado del Bienestar, sobre todo con su intervención en la educación. Y sobre todo a través de la escuela pública. Actualmente, las referencias neoliberales y neoconservadoras nos han llevado a procesos que conllevan que la igualdad de oportunidades, principio de referencia en el sistema educativo español, se convierta en procesos de segregación (Bernal y Lorenzo, 2012).

Uno de esos procesos es la elección de centro escolar por parte de las familias, que en teoría se debería materializar mediante mecanismos que favorecieran el acceso igualitario, con independencia de todo tipo de condicionantes naturales o socioeconómicos. Sin embargo, se puede considerar a España como uno de los países con el sistema educativo más segregador de toda la Unión Europea (Murillo y Martínez-Garrido, 2018). Esto se concreta en que las diferentes escuelas acogen de manera desigual a alumnos con diferentes perfiles socioeconómicos y culturales y, por tanto, en vez de constituirse en escenarios idóneos para la cohesión, las escuelas se convierten en espacios donde se reproducen o se refuerzan las desigualdades sociales.

El sistema educativo español se organiza fundamentalmente como un servicio público y, como tal, garantiza el derecho y la obligatoriedad de la educación (Constitución Española, artículos 27.1, 27.4). Así, todas las familias tienen el derecho y la obligación de enviar a sus hijos a un centro escolar. Estableciendo el sistema que sean las familias las que escojan libremente el centro de su preferencia. Esta libertad otorgada a las familias ha permitido la promoción y adopción de políticas de mercado en el campo de la educación convirtiendo a las escuelas en un vasto campo de competición (Laval, 2004, p. 136; Bernal, 2005), y a los padres en "usuarios" con libertad para elegir entre distintos "proveedores". En este sentido, muchas voces críticas advierten del riesgo que supone esta libertad de elección para la igualdad de oportunidades teniendo en cuenta que para llevar a cabo una elección real el acceso a la información es clave y las familias con mayor poder adquisitivo y mayor nivel formativo disponen de más recursos para acceder a ella, por lo tanto, la libertad de elección puede ser en una peligrosa trampa (Torres, 2001; Bernal, 2005).

Diversos estudios realizados en España han demostrado que tanto los factores familiares como el nivel de ocupación y nivel de estudios de los padres, así como la dotación de bienes y recursos económicos, la dotación de bienes y recursos culturales, el país de origen, la estructura y el tamaño de la familia, son factores que explican la distribución de los estudiantes en los diferentes tipos de centros (Villarroya, 2002; Fuenmayor, Granell y Villarreal, 2003; Albert y García-Serrano, 2004; Bernal, 2005; Villamar, 2005; Olmedo, 2007; Mancebón-Torrubia y Ximénez-de-Embún, 2007; Urquizo, 2008; Escardíbul y Villarroya, 2009a). Así, los hijos de inmigrantes y alumnos que provienen de hogares con menos capital económico, social y cultural están más representados en centros públicos y, dentro estos, en un espectro concreto; y los alumnos cuyos padres poseen mejores trabajos y mayores niveles de estudios y disponen de una mayor dotación de bienes y recursos culturales, están más representados en centros concertados o privados. "Se concluye que los mecanismos de mercado responden a un plan para configurar la red escolar pública como subsidiaria de la red privada. Además, se evidencia que la mayoría de familiares no tiene capacidad para elegir determinados centros, por lo que principios como la igualdad, equidad y justicia social quedan en entredicho" (Luengo y Molina, 2018)

Esta polarización del alumnado en los diferentes tipos de centros (Alegre, Benito y González, 2010) provoca una brecha muy importante en el rendimiento de los estudiantes según la titularidad de centro, siendo los alumnos de los centros concertados y privados los que suelen obtener mejores resultados. (Mancebón-Torrubia, y Ximénez- de-Embún, 2010; Choi y Calero, 2012).

Las políticas que se desarrollan para garantizar la libre elección en las distintas comunidades no son las mismas. Hay comunidades donde se aboga por una mayor libertad para los padres, evidentemente son comunidades gobernadas por partidos políticos afines a un sistema neoliberal de organización social. Por ejemplo, la Comunidad de Madrid apuesta por el distrito único, lo que permite a los padres elegir el centro que quieran, vivan donde vivan, provocando que las escuelas 
compitan entre sí porque están inmersas en una lógica de mercado. Lógicamente, esto no facilita una competición de igual a igual entre un centro público y otro concertado.

En el extremo opuesto están las comunidades autónomas donde se desarrollan políticas caracterizadas por la regulación y gestión pública del proceso de elección. Un ejemplo lo tenemos en la Comunidad de Aragón donde se apuesta por la zonificación escolar múltiple, un tipo de regulación basada en la priorización del criterio de proximidad.

En cualquier caso, hay que destacar que, si bien el distrito único per se no es segregador, hay evidencias de que es favorable para las familias con mayor poder adquisitivo (Murillo y MartínezGarrido, 2018) y la zonificación escolar múltiple, tal como se plantea, tampoco es un mecanismo eficaz para garantizar un sistema educativo equitativo.

El presente artículo tiene como objetivo aportar más evidencias acerca de los efectos de la libre elección en la igualdad de oportunidades en el acceso a un centro escolar y, por tanto, en la equidad del sistema educativo, a partir de las experiencias y discurso de las propias familias.

\section{Método}

Este trabajo es parte de un estudio empírico más amplio realizado en Zaragoza durante los años 2013-2015, titulado La elección de centro escolar en Aragón: Razonamientos y estrategias de los padres en la elección de centro escolar. Esta investigación tenía como propósito averiguar el significado que adquiere para las familias aragonesas, en concreto de la ciudad de Zaragoza, la elección de centro escolar. Con qué expectativas participan los padres en el proceso de elección, qué tipo de razonamiento aplican, cuáles son sus posibilidades y sus limitaciones, qué estrategias ponen en marcha para acceder al centro de su preferencia y qué niveles de satisfacción experimentan.

La población de estudio estaba conformada por familias con hijos matriculados en $2^{\circ}$ curso de Educación Infantil, en centros públicos, concertados y privados de las siete zonas escolares de la ciudad de Zaragoza, durante el año lectivo 2013-2014.

Los datos fueron obtenidos mediante una encuesta en la que participaron 1837 familias, y a través de 43 entrevistas en profundidad, 27 de ellas a padres que formaron parte de la encuesta y 16 a otros agentes educativos locales (siete directores de colegios públicos, dos directores de colegios concertados, dos directores de colegios privados, un inspector provincial de educación, un representante de asociaciones de padres y madres de centros públicos y otro de centros concertados, un representante sindical y un representante de la Comunidad Islámica de Zaragoza).

Los padres que participaron en estas entrevistas fueron seleccionados teniendo en cuenta cinco criterios: la composición social del centro donde asisten sus hijos (centros de barrios ricos y centros de barrios más modestos de la ciudad); el nivel de demanda de plazas en el centro donde asisten sus hijos (centros donde faltan plazas y centros donde sobran plazas); tipo de centro donde asisten sus hijos según su titularidad y fuente de financiación (centros públicos, concertados y privados); el país de origen de los padres (familias españolas y familias extranjeras) y la zona de escolarización (familias de las diferentes zonas escolares de Zaragoza). Estas entrevistas fueron realizadas a lo largo del segundo cuatrimestre del curso 2013 - 2014. Los resultados de estas fueron analizados siguiendo los principios de la Teoría Fundamentada (Strauss y Corbin, 2002).

Para los efectos de este artículo se han utilizado de manera preferente los datos obtenidos a través de las entrevistas en profundidad. Lógicamente, como las entrevistas se realizaron después de los cuestionarios, las líneas del discurso que se plantea emanan también de los datos de las entrevistas.

\section{Resultados}

Como ya hemos comentado, se trataba de analizar el discurso de las familias para identificar aquellos aspectos de la libertad de elección que en la práctica tienen efectos en la igualdad de oportunidades. De los resultados se deduce que hay tres factores que determinan la participación de 
los padres en el proceso de elección: amplitud, diversidad y calidad de la oferta educativa. La amplitud de la oferta educativa como señala Andrada (2008), se refiere a la cantidad de centros que hay en un espacio local concreto; la diversidad se refiere a la existencia de más de un tipo de centro escolar elegible y la calidad es entendida por los padres como las condiciones en que se desarrollan los procesos de enseñanza aprendizaje que a su vez afectan los logros académicos de los estudiantes.

En relación con la amplitud de la oferta educativa, una de las primeras observaciones que hacen las familias es obvia, para participar en un proceso de elección de centro escolar, lo fundamental es tener alternativas diversas entre las cuales elegir; si no es así, la libertad de elección tiene poco sentido. Por ello, la libertad de elección es un derecho que se circunscribe en los grandes núcleos urbanos y, por lo tanto, los padres que residen en pueblos pequeños o núcleos rurales no eligen.

Pero también en los grandes núcleos urbanos, como lo es Zaragoza, dependiendo de la zona de residencia de los padres, estos tienen más o menos opciones para elegir. Los que viven en los barrios nuevos encuentran una dificultad añadida, ya que el número de plazas escolares es insuficiente para atender a toda la demanda local. Esta situación obliga a muchos padres a desplazarse a otras zonas de la ciudad, asumiendo los gastos adicionales que conlleva el desplazamiento (transporte, comedor, etc.). Desde esta perspectiva, y a falta de medidas compensatorias, el sistema es considerado por muchas familias como un sistema injusto.

[...] si te obligan a ir a un colegio que está lejos, a lo mejor te están obligando a asumir el gasto de comedor. Yo estoy gastando 100 euros por cada bijo y si mi bijo comiera en casa seguramente no gastaría 100 euros. Entonces no es justo en ese sentido. (Madre española de Colegio Público. Entrevista No 6)

En cuanto a la diversidad de la oferta educativa (que tampoco existe en el medio rural), en las zonas urbanas, y particularmente en el municipio de Zaragoza, hay una distribución bastante desigual de los distintos tipos de centros en los diferentes distritos o barrios del término municipal. Hay distritos donde existe una diferencia muy amplia, en cuanto a número de centros públicos o concertados. Por ejemplo, en Zaragoza las familias que viven en el distrito Centro tienen un margen muy reducido para optar por un colegio público, debido a que la mayoría de los centros son concertados (de los trece centros que hay en dicho distrito, diez son concertados). Ocurre lo contrario en otros distritos como El Rabal, donde los padres tienen menos posibilidades de escolarizar a sus hijos en un centro concertado, porque de los catorce colegios que hay en el distrito, doce son públicos y solo dos son concertados. Circunstancias como estas, junto con el mecanismo de adjudicación de centro poco flexible, ya que otorga mucha importancia a la proximidad del domicilio familiar o laboral del solicitante, perjudican a muchas familias en su libertad de elección.

[...] yo soy alemana y con la niña bablamos alemán en casa. Yo quería que ella pudiera aprender a escribiry leer también en alemán. Por tanto, mi primera preferencia era un colegio bilingüe alemán, el Colegio Público E. G. que está en la Romareda. Y como no vivimos en esa zona, tampoco trabajo en esa zona, no teníamos ninguna posibilidad de llevar a mi hija abí. [...] También hay otro colegio público bilingüe en alemán, el Colegio C. A., pero también ese está en la otra punta de la ciudad y luego hay el Colegio Alemán que es totalmente privado. [...] Si tienes un poder adquisitivo alto se te amplian las opciones y te permites arriesgar más. (Entrevista $\mathrm{N}^{\mathrm{o}} 2$ )

En cuanto a la calidad de la enseñanza, la mayoría de los padres consideran que los colegios que conforman las redes escolares en Zaragoza tienen diferentes niveles de calidad. Entre los colegios públicos hay algunos mejor valorados que otros, y los colegios concertados generalmente reciben mejor valoración que los públicos.

Yo creo que es tan obvio que la Administración tiene que estar para igualar los colegios públicos, pero igualar en todos los sentidos. No puede ser que un niño que vive aqui tenga una calidad de educación y por vivir tres kilómetros más allá, tenga otra. [...] si tú les equiparas en recursos y en vez de decir, como se supone que se va a hacer con las pruebas externas, "este colegio tiene unos profesores maravillosos, los críos sacan unas notas altísimas, éste lo está haciendo muy bien, toma, más dinero". No, perdona, es que es al revés, es que estáis haciendo al revés. (Madre española de Colegio Público. Entrevista No 21) 
Los padres entienden que en teoría los programas curriculares son comunes en todos los centros públicos. Pero, añaden, no todos los colegios tienen una población escolar equiparable en términos de diversidad. En muchos colegios difícilmente se pueden llevar a cabo procesos de enseñanza aprendizajes "normalizados".

Según los padres hay dos circunstancias que determinan la composición del alumnado en los colegios sostenidos con fondos públicos de Zaragoza: la primera es la ubicación del centro y la segunda, la titularidad de este. Es decir, el perfil socioeconómico y cultural del alumnado de un colegio depende del barrio en que este está ubicado. Por poner un ejemplo, en Zaragoza no es lo mismo un colegio público de Torrero que otro de la Romareda.

En el Colegio E. G. (de la Romareda) hay 12 niños con becas de comedor, en mi centro (de Torrero) hay ahora mismo 132, la desproporción es salvaje. Eso significa que en mi colegio hay 132 familias que están por debajo de los siete mil y pico de euros al año, eso es una barbaridad porque es el umbral de la miseria. [...] no es lo mismo, porque cuando en una familia entran 7.500 euros al año, tiene que haber problemas de narices, alli estás rozando lo básico y como decía mi abuela, contigo pan y cebolla es mentira. Hace falta un cierto nivel económico para que los problemas no se conviertan en dramas, porque yo creo que están muy relacionados el nivel económico y los problemas estructurales. (Madre española de Colegio Público. Entrevista No 16)

Tampoco es lo mismo un centro público que uno concertado, en cuanto a la composición de su población escolar, aunque estén en el mismo barrio. Los alumnos de origen inmigrante y pertenecientes a minorías étnicas, que, por lo general, provienen de familias con menor capital económico, social y cultural, están concentrados mayoritariamente en los centros públicos. Sin embargo, los colegios concertados tienen un alumnado más homogéneo.

Existe una percepción generalizada entre las familias de que la excesiva presencia en un mismo centro de alumnos que provienen de situaciones sociales y culturales desfavorecidas, repercute negativamente en la calidad de la enseñanza. Esta es una de las razones principales por las que padres autóctonos, en la medida de lo posible, tratan de evitar estos tipos de colegios que por lo general son públicos, produciéndose un trasvase de alumnos a los concertados cuya población escolar se compone mayoritariamente de niños españoles. Para las familias que buscan esta diferenciación, los centros concertados se convierten en alternativas hechas a su medida. Las siguientes entrevistas son claramente reveladoras.

Yo conozco desde gente que a lo mejor vive por ..., y que les correspondería los colegios donde casi no hay niños españoles y para evitar eso, lo primero que hacen es ir a los colegios concertados aunque no tengan ninguna fe religiosa, ni ningún interés religioso, prefieren llevar a los niños a colegios religiosos porque claro, los colegios concertados son privados aunque estén subvencionados y si que requieren ya una cierta capacidad económica, hay que comprar uniformes, las extraescolares, el comedor más caro, a veces el transporte... lo primero, que van a estos colegios aunque no sea por motivo religioso. (Madre española de Colegio Público. Entrevista No 4)

[...] si este colegio [Colegio Público C. A.] fuera como cuando yo era pequeña, que habia pocos inmigrantes, a la gente no le importaría venir a este colegio, por lo que les molesta venir a muchos de ellos, es porque hay muchos inmigrantes y no se quieren mezclar. (Madre española de Colegio Público. Entrevista No 6)

[...] yo creo que muchos van a los concertados porque quieren hacer una limpieza étnica. "Yo voy a concertado así me evito a los musulmanes, porque los concertados son casi todos religiosos, me los quito. Ya sólo me quedan los rumanos, los sudamericanos, o sea, que como hay que pagar una cuota mensual seguro que no es barato para hacer frente a esa cuota, seguro que vamos a quedar, pues los rico". Como para mi eso es hacer una apología de una especie de casta, creo que no hace ningún bien al niño... (Padre español de Colegio Público. Entrevista $\mathrm{N}^{\mathrm{o}}$ 5)

Es necesario destacar que participar en el proceso de elección descartando colegios que no gustan, no todas las familias lo pueden hacer. Los datos obtenidos a través de las encuestas indican que: 
1. Nueve de cada diez familias que han elegido centros públicos y cuyo nivel de renta mensual es superior a $3000 €$ afirman que están en este tipo de centro porque realmente tienen preferencia por la enseñanza pública. Sin embargo, tres de cada diez familias con rentas mensuales inferiores a $1000 €$ o sin ingreso han matriculado a sus hijos en centros públicos a pesar de que tenían preferencia por la educación concertada o privada. (Gráfico 1).

2. El $86 \%$ de los padres que poseen estudios universitarios y llevan a sus hijos a centros públicos han elegido este tipo de centro porque se corresponden con su preferencia real. Sin embargo, tres de cada diez padres que solo cuentan con estudios obligatorios o menos y llevan a sus hijos a centros públicos hubieran preferido la enseñanza concertada o privada. (Tabla 1).

3. El $84 \%$ de las familias españolas y el $81 \%$ de las familias mixtas que están en un centro público han escogido este tipo de centro porque realmente tienen preferencia por la educación pública. Sin embargo, el 35\% de las familias extranjeras que han optado por un centro público hubieran preferido escolarizar a sus hijos en un centro concertado o privado. (Gráfico 2).

Gráfico 1

Preferencia por centros públicos según nivel de renta familiar

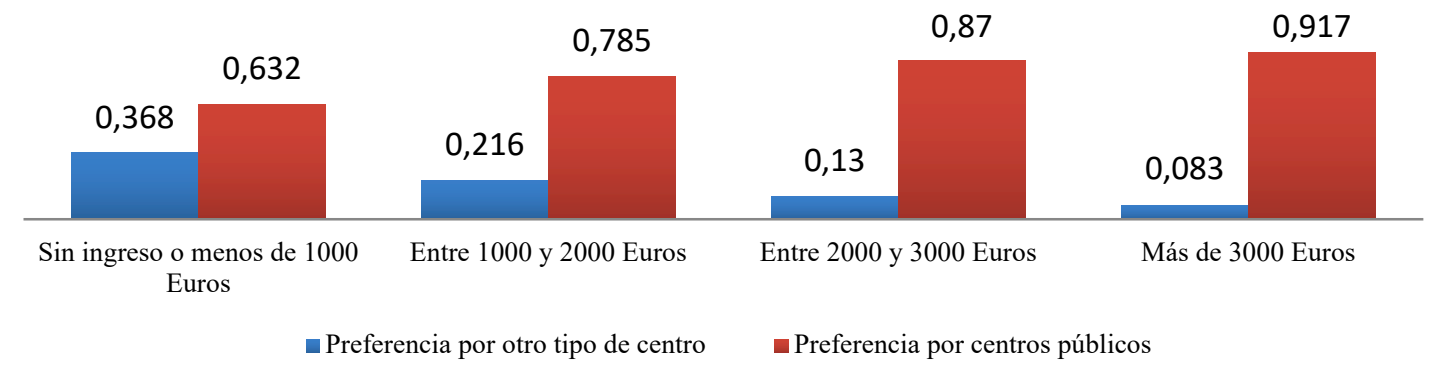

$X^{2}(3)=73,481 p<0.05$

Tabla 1

Preferencia por centros públicos según nivel de estudios de los padres.

\begin{tabular}{lcc}
\hline Nivel de estudios de los padres & $\begin{array}{c}\text { Preferencia por otro tipo } \\
\text { de centro }\end{array}$ & $\begin{array}{c}\text { Preferencia por centros } \\
\text { públicos }\end{array}$ \\
\hline Sin estudios o primarios incompletos & $30,4 \%$ & $69,6 \%$ \\
Primarios o secundarios obligatorios & $27,8 \%$ & $72,2 \%$ \\
Postobligatorios no universitarios & $23,8 \%$ & $76,2 \%$ \\
Estudios universitarios & $13,8 \%$ & $86,2 \%$ \\
\hline
\end{tabular}

$X^{2}(3)=29,530 p<0.05$

Gráfico 2

Preferencia por centros públicos según tipo de familia

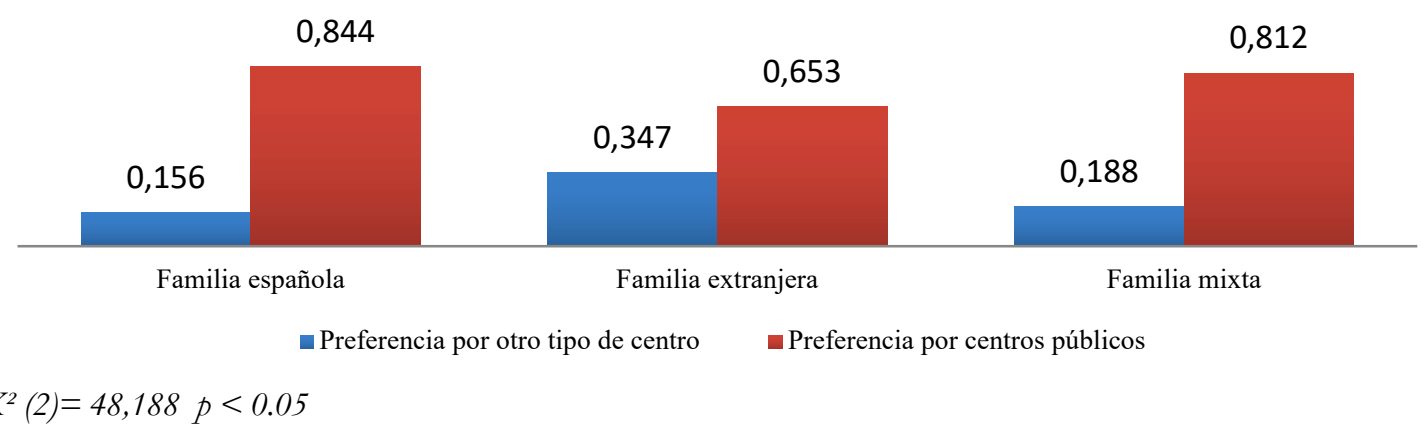


A partir de estos resultados se puede deducir que en el contexto educativo local los padres autóctonos, con más dinero y nivel de estudios más alto, tienen mayor probabilidad de estar en el colegio de su preferencia real. Por el contrario, tanto los inmigrantes como aquellos padres que disponen de menos estudios y menores recursos económicos, tienen escaso margen de elección y, por tanto, tienen mayor probabilidad de acabar escolarizando a sus hijos en centros públicos más cercanos a su domicilio, aunque este tipo de centros no se corresponda con su preferencia real.

Se produce una situación de desigualdad en las oportunidades ya en el inicio de la educación en el sistema educativo. Existe un colectivo de familias que por disponer de mayor renta o nivel cultural pueden elegir y otras familias a las que el sistema les niega esa posibilidad. La situación cada vez mayor de guetos educativos es ciertamente alarmante. Según el último informe del Ministerio de Educación y Formación Profesional (2018-19), en el curso 2016-17 el alumnado extranjero estaba escolarizado en un $79,5 \%$ en los centros públicos, siendo solamente el 14,8 en los concertados y el 6,7 en los privados, lo que constituye un dato irrefutable de la desigualdad en el inicio de la escolaridad.

En bastantes ocasiones, según los padres, la política de admisión en los colegios concertados supone un filtro para muchas familias, especialmente para las que proceden de entornos más desfavorecidos, que es el caso de la mayoría de los inmigrantes, pero también para los gitanos y alumnos con necesidades educativas especiales. El uso del uniforme, el pago de una cuota voluntaria, el coste de las actividades extraescolares, del comedor, etc., son gastos que muchas familias no pueden solventar, por lo que, a pesar de ser un tipo de centro sostenido con fondos públicos, están fuera de sus opciones a la hora de elegir. En este caso, los padres saben, pero no pueden ejercer su libertad de elección.

Lo que ocurre es que los colegios concertados ponen tantos filtros que acaban seleccionando mucho, [...] Tü ves el indice de penetración de inmigración en los colegios públicos y los concertados y es salvaje la diferencia. Algo está pasando. Estudiar en un colegio concertado se puede, pero bay que pagar un chándal, los materiales, los libros que son más caros, entonces uno del nivel medio-bajo no puede, no llega o hay que prescindir de muchas cosas para llegar...Todo eso ya es un filtro en la entrada. (Madre española de Colegio Público. Entrevista No 16)

Es por los solicitantes, los solicitantes de un colegio concertado no son lo mismo que los solicitantes de un colegio público. Y luego abí bay una división social y económica, los concertados no son exactamente gratuitos, es gratuita a lo mejor lo que es la enseñanza reglada pero luego hay una serie de gastos que les cobran y en el público no. [...] Porque luego hay personas que se manifiestan ateas o agnósticas o no sé qué, pero luego envían a sus hijos a un concertado. Hasta bay gente musulmana que lleva a su bijo a un concertado. El ideario yo creo que no, es más bien por una razón socioeconómica que no sé si tiene nombre. (Padre español de Colegio Público. Entrevista $\mathrm{N}^{\mathrm{o}} 12$ )

A las familias que buscan la diferenciación hay que sumarles aquellas que optan por los colegios concertados porque proyectan un nivel social y una gestión escolar más eficaz. Los padres consideran que en estos centros hay una mayor "disciplina" y una mayor exigencia en términos académicos, y, como consecuencia, los alumnos salen mejor preparados para los estudios de etapas o niveles superiores; y, por lo tanto, perciben que hay mayor garantía de calidad en este tipo de centros.

Los resultados son diferentes dependiendo del colegio, depende de muchisimos factores, depende de los niños, depende de los profesores, depende de que en cada colegio rige unas normas internas... No sabes cómo será el futuro de tus bijos, pero si que puedes saber que en este colegio cuando terminan, pues tienen un plus para el tema de selectividad y esas cosas, tienen un poquito... [...] [Los colegios concertados] te ofrecen más garantía. (Padre español de Colegio Concertado. Entrevista $N^{\circ} 7$ )

\section{Discusión}

El proceso de elección de centro es esencial para conseguir el centro escolar mejor valorado y evitar aquel que, en teoría, se piensa que no es bueno. Es decir, las familias practican lo que Alegre y Benito (2012) denominan elección en positivo y elección en negativo. 
Sin embargo, esta forma de proceder de los padres está fuertemente condicionada por la capacidad individual de cada familia, en términos socioeconómico y cultural. Así, los padres con mayor poder adquisitivo y mayor nivel de instrucción tienen márgenes de elección más amplios, están mejor informados y pueden optar por el colegio que tienen cerca si lo consideran bueno; tienen recursos para arriesgar y optar por el colegio que está fuera de su entorno si ninguno de los que están cerca se ajusta a lo que buscan y, en últimas instancias, pueden optar por un colegio totalmente privado. Como consecuencia, estas familias están distribuidas, por lo general, en los colegios que se corresponden con su verdadera preferencia. Por el contrario, las familias con menos poder adquisitivo y menos nivel de instrucción optan principalmente por el centro donde tienen prioridad por cercanía, independientemente de que este se corresponda con lo que realmente desean.

Por lo tanto, hay familias que se benefician del proceso de elección y otras que juegan con desventajas, en definitiva, hay familias que eligen y otras que no (Fernández Esquinas, 2004). Las primeras son las que están en los colegios donde realmente quieren estar, y las segundas son las que con su participación en el proceso de elección están legitimando un mecanismo que propicia y normaliza la existencia de escuelas que reproducen las mismas desigualdades que hay en la sociedad.

Las familias consideran que esta estrategia de huida de muchos padres hacia los colegios mejores favorece a los concertados porque consiguen un alumnado mucho más homogéneo en comparación con la población escolar de los colegios públicos. Esta circunstancia es lo que hace que los colegios concertados sean más demandados por las familias, a pesar de que la escolarización en ellos supone un gasto adicional para los padres, duplicando lo que cuesta la escolarización en centros públicos (Hidalgo, 2005; Rogero-García y Andrés-Candelas, 2014). Fernández y Muñiz (2012) hablan de un círculo vicioso de la escuela concertada que origina los procesos de segregación social y, en consecuencia, la pérdida de equidad y de igualdad de oportunidades en nuestro sistema educativo.

Según Choi y Calero (2012), existe una brecha muy importante en el rendimiento de los estudiantes según la titularidad de centro, siendo los alumnos de los centros concertados y privados los que obtienen mejores resultados. De acuerdo con Mancebón-Torrubia, y Ximénez- de-Embún (2010), la explicación de dicha diferencia la encontramos en la composición socioeconómica de los centros. En los centros públicos están sobrerrepresentados los alumnos que provienen de hogares más desfavorecidos e inmigrantes. Y al revés, en los centros concertados y privados asisten alumnos que en general pertenecen a familias con un estatus socioeconómico y cultural más alto. Esta desigualdad de partida favorece a los centros privados y concertados en cuanto a logros educativos.

Bastantes estudios coinciden en señalar que, una vez que se controlan las variables relacionadas con el origen socioeconómico de los alumnos, no existen diferencias significativas entre los resultados académicos de los distintos tipos de centros que configuran las redes escolares en España (Calero y Escardíbul, 2007; Anghel y Cabrales, 2010; Perelman y Santín, 2011; Cordero, Manchón y Simancas, 2012; Choi y Calero, 2012). Incluso los centros públicos llegan a obtener mejores resultados (Urquizu, 2008; Mancebón-Torrubia, Calero, Choi y Ximénez-de-Embún, 2012). Esto nos lleva a decir que si se planteara otro sistema de elección de centro, similar por ejemplo al establecido en California, en el que los alumnos tienen que ir a aquel centro que les corresponde por la vivienda (cada zona tiene un solo centro de referencia) y no, como en España, donde las zonas de influencia son muy amplias, se lograría evitar la falta de equidad de inicio que vamos destacando en la tesis de este artículo. Otra cuestión ya sería que las zonas en sí mismas tuvieran esa falta de equidad.

\section{Conclusiones}

Los padres son libres de elegir el centro escolar que consideren más apropiado para la educación formal de sus hijos, es un principio constitucional. Sin embargo, tal como hemos podido constatar en este estudio, no todos los padres pueden hacer efectivo ese derecho (los que residen en zonas rurales o en municipios pequeños, no eligen); ni aquellos que pudiendo participar en la elección lo hacen en igualdad de condiciones.

Hay dos factores que condicionan fuertemente la participación de las familias en la elección de centro: el nivel de formación de los padres y la renta familiar. Así, los que tienen más tienen más 
oportunidades para elegir, y al revés, las oportunidades se reducen para los que tienen menos. Esta relación positiva tiene nombre: desigualdad.

Por lo tanto, se puede considerar el sistema de elección de centro como otro mecanismo que va estructurando de forma subliminal un modelo en el que la denominada libertad de elección de centro escolar se convierte en una forma de segregación y desigualdad. Así, se va aumentando las zonas de escolarización, teniendo como meta el establecimiento de zonas únicas, que, según los planteamientos neoliberales, permiten una mayor libertad en la elección de centros. Lo que no se dice es que aumentan los colegios elegibles, pero no "accesibles" para todas las familias. La tesis de que este modo de elección favorece la competitividad entre los centros y la libertad de elección individual de los padres queda claramente desmontada con diversos estudios e investigaciones llevadas a cabo en los últimos años (Ball, 1993; Carabaña, 1985; Van Zanten; 2005; Bernal, 2005; Olmedo 2007, 2012; Alegre, 2010; Mancebón- Torrubia y Ximénez-de-Embún, 2007, 2008, 2010, 2014; Fernández Enguita, 2008; Escardíbul y Villarroya, 2009b; Crespo, Díaz y Pérez, 2012; Bernal y Lorenzo, 2012; Capellán del Toro, García, Olmos y Rubio, 2013; Murillo y Martínez-Garrido, 2018). Estos estudios señalan que la libertad de elección per se no garantiza una igualdad de oportunidades y que, si no va acompañada de políticas que busquen reducir las desigualdades de partida que hay entre las familias, la libertad de elección funciona como un mecanismo de segregación social. Esto nos lleva a una consideración más compleja, ya que tendríamos que hablar de políticas sociales que pudieran provocar una menor desigualdad social, algo que en las reglas de juego neoliberales no se contempla.

\section{Referencias bibliográficas}

Albert, C. y García-Serrano, C. (2004). Tipo de escuela y logros educativos en España. Comunicación presentada en el XIII Congreso de la Asociación de Economía de la Educación. San Sebastián, España. https://bit.ly/2luO7Xt

Alegre, M. A., Benito, R. y González, I. (2010). Measures and Determinants of Student Body Socioeconomic Diversity: Evidence from Spain. Journal of School Choice, 4 (1), 23-46. https://doi.org/10.1080/15582151003626343

Alegre, M.A. (coord.) (2010). Les families davantl'elecció escolar. Dilemes i desigualtats en la tria de centre a la ciutat de Barcelona. Barcelona: Fundació Jaume Bofill

Alegre, M. A. y Benito, R. (2012). ¿En qué se fijan las familias a la hora de escoger la escuela de sus hijos? Factores de elección y descarte escolar en la ciudad de Barcelona. Profesorado: Revista de currículum y formación del profesorado, 16(3), 59-79. https://bit.ly/2lCMfeZ

Andrada, M. (2008). Libertad de elección escolar, mecanismos de atribución de plazas y preferencias familiares: una evaluación a partir de criterios de equidad. Profesorado: Revista de currículum y formación del profesorado, 12(2). https://bit.ly/2lBpkAM

Ball, S. J. (1993). Education markets, choice and social class: the market as a class strategy in the UK and USA. British Journal of Sociology of Education, 14(1), 3-20. https://doi.org/10.1080/0142569930140101.

Bernal, J. L. (2005). Parental choice, social class and market forces: The consequences of privatization of public services in education. Journal of Education Policy, 20 (6), 779-792. https://doi.org/10.1080/02680930500293825

Bernal, J. L. y Lorenzo, J. (2012). La privatización de la educación pública. Una tendencia en España. Un camino encubierto hacia la desigualdad. Profesorado: Revista de currículum y formación del profesorado, 16 (3), 81-109. https://bit.ly/2lxXG81 
Cabrales, A y Anghel, B. (2014). The Determinants of Success in Primary Education in Spain. Revista de Evaluación de Programas y Politicas Públicas, 2, 22-53 https://doi.org/10.5944/reppp.2.2014.12030

Calero, J. y Escardíbul, J. O. (2007). Evaluación de servicios educativos: el rendimiento en los centros públicos y privados medido en PISA-2003. Hacienda Pública Española, 183, 33-66. https://bit.ly/2k3qjtk

Capellán del Toro, L., García, F. J., Olmos, A. y Rubio, M. (2013). Sobre agrupamiento, concentración, segregación o guetización escolar: Claves para un análisis interpretativo de tales situaciones y procesos. Avances en Supervisión Educativa: Revista de la Asociación de Inspectores de Educación de España, 18. https://bit.ly/2khxTAN

Carabaña, J. (1985). Informe sobre el estudio referente a la elección de centro de enseñanza por los padres. Madrid: CIDE

Choi, A. y Calero, J. (2012). Rendimiento académico y titularidad de centro en España. Profesorado: Revista de curriculum y formación del profesorado, 16(3), 31-57. https://bit.ly/2IXRdmX

Cordero, J., Manchón, C. y Simancas, R. (2012). Análisis de los condicionantes del rendimiento educativo de los alumnos españoles en PISA 2009 mediante técnicas multinivel. Presupuesto y Gasto Público, 67, 71-96. https://bit.ly/2lXYcMH

Crespo, E., Díaz, C. y Pérez, J. (2012). Factores condicionantes de la desigualdad educativa: Un análisis para el caso español. En Pacheco, E. (Coord.) Investigaciones de Economía de la Educación, 7, 759-774. https://bit.ly/2lu5FTr

Decreto 32/2007, de 13 de marzo, por el que se regula la admisión de alumnos en los centros docentes públicos y privados concertados en las enseñanzas de segundo ciclo de educación infantil, educación primaria, educación especial, educación secundaria obligatoria, bachillerato y formación profesional de la Comunidad Autónoma de Aragón. BOA núm. 31, de 14 de marzo de 2007, pp. 4077 - 4087.

Escardíbul, J. O. y Villarroya, A. (2009a). Determinantes de la elección de centro escolar. En J. M. Roig y L. E. Vila (Coords), Investigaciones de Economía de la Educación, 4, 159-169. https://bit.ly/2lXNSnY

Escardíbul, J. O. y Villarroya, A. (2009b). Desigualdades en la elección de centro escolar en España. Comunicación presentada en el XVI Encuentro de Economía Pública. Granada, España. https://bit.ly/2ksbxMQ

Fernández Enguita, M. (2008). Escuela pública y privada en España: La segregación rampante. Profesorado: Revista de curriculum y formación del profesorado, 12 (2). https://bit.ly/2kqJjSF

Fernández Esquinas, M. (2004). Elección de escuela: efectos sociales y dilemas en el sistema educativo público en Andalucía. Revista de Educación, 334, 377-390. https://bit.ly/2luZ5fB

Fernández, R. y Muñiz, M. (2012). Colegios concertados y selección de escuela en España: un círculo vicioso. Presupuesto y Gasto Público, 67, 97-118. https://bit.ly/2lwRQUg

Fuenmayor, A., Granell, R. y Villarreal, E. (2003). Determinantes en la elección de centro educativo por parte de los padres. Estudios de Economía Aplicada, 21 (2), 377- 389. https://bit.ly/2ktKkJx

Hidalgo, I. (2005). Gasto de las familias en educación básica y elección entre colegio público y privado: un análisis empírico. Tesina CEMFI No 0504. Madrid: Centro de Estudios Monetarios y Financieros. ftp://ftp.cemfi.es/mt/05/t0504.pdf 
Laval, Ch. (2004). La escuela no es una empresa: el ataque neoliberal a la enseñanza pública. Barcelona: Paidós.

Luengo, J. y Molina J. (2018). (Re)-contextualización del modelo de cuasi-mercado en Andalucía. Dirección escolar y familias compitiendo y seleccionando. Revista de $\begin{array}{llll}\text { Curriculum } y \text { Formación del Profesorado, } & \text { 22(2). }\end{array}$ https://recyt.fecyt.es/index.php/profesorado/article/view/66386

Mancebón-Torrubia, M. J. y Ximénez-de-Embún, D. P. (2007). Conciertos educativos y selección académica y social del alumnado. Hacienda Pública Española, 180, 77- 106. https://bit.ly/2kqSbYr

Mancebón-Torrubia, M. J. y Ximénez-de-Embún, D. P. (2008). El alumnado de los centros educativos aragoneses de enseñanza secundaria: segregación socioeconómica y académica por tipo de centro y percepciones de la calidad educativa. Revista de Economía Aragonesa, 35, 115-136. https://bit.ly/2jUqW8i

Mancebón-Torrubia, M. J. y Ximénez-de-Embún, D. P. (2010). Una valoración del grado de segregación socioeconómica existente en el sistema educativo español. Un análisis por Comunidades Autónomas a partir de PISA 2006. Regional and Sectorial Economic Studies, 10 (3), 129-148. https://bit.ly/21CK7nv

Mancebón-Torrubia, M. J., Calero, J., Choi, A. y Ximénez-de-Embún, D. P. (2012). The efficiency of public and publicly subsidized high schools in Spain. Evidence from PISA 2006. Journal of the Operational Research Society, 63(11), 1516-1533. https://doi.org/10.1057/jors.2011.156.

Mancebón-Torrubia, M. J. y Ximénez-de-Embún, D. P. (2014). Equality of school choice: a study applied to the Spanish region of Aragón. Education Economics, 22(1), 90-111. https://doi.org/10.1080/09645292.2010.545197.

Ministerio de Educación y Formación Profesional (2018). Datos y cifras. Curso escolar 20182019. Madrid, Secretaría General Técnica

Murillo, F. Javier y Martínez-Garrido, C. (2018). Magnitud de la segregación escolar por nivel socioeconómico en España y sus Comunidades Autónomas y comparación con los países de la Unión Europea. Revista de Sociología de la Educación (RASE), 11(1), 3758. https://doi.org/10.7203/RASE.11.1.10129.

Olmedo, A. (2007). Las estrategias de elección de centro educativo en las familias de clase media. Estudio de la incidencia social en un mercado educativo local. [Tesis doctoral]. Universidad de Granada, Facultad de Ciencias de la Educación, Granada.

Olmedo, A. (2012). ¿Más información, más libertad y más igualdad conllevan mejores resultados? Elección escolar y desigualdades educativas. Cuadernos de Pedagogía, 425, 38 42.

Perelman, S. y Santín, D. (2011). Measuring Educational Efficiency at Student Level with Parametric Stochastic Distance Functions: an Application to Spanish PISA Results. Education Economics, 19 (1), 29-49. https://doi.org/10.1080/09645290802470475.

Rogero-García, J. y Andrés-Candelas, M. (2014). Gasto público y de las familias en educación en España: diferencias entre centros públicos y concertados. Revista Española de Investigaciones Sociologicas, 147, 121-132. https://doi.org/10.5477/cis/reis.147.121.

Strauss, A. y Corbin, J. (2002). Bases de la investigación cualitativa. Técnicas y procedimientos para desarrollar la teoría fundamentada ( $1^{\mathrm{a}}$ ed. en español; E. Zimmerman, Trad.). Medellín: Editorial Universidad de Antioquia. (Trabajo original publicado en 1998).

Torres, J. (2001). Educación en tiempos de Neoliberalismo. Madrid: Morata. 
Urquizo, I. (2008). La selección de escuela en España. RASE: Revista de la Asociación de Sociología de la Educación, 1 (2), 70-89. https:// bit.ly/2IXNKEX.

Van Zanten (2005). New modes of reproducing social inequality in education: the changing role of parents, teachers, schools and educational policies. European Educational Research Journal, 4(3), 155-169. https://doi.org/10.2304/eerj.2005.4.3.1.

Villamar, P. (2005). La libertad de elección en educación: Análisis pedagógico de la situación nacional e internacional. [Tesis doctoral]. Universidad Complutense, Facultad de Educación, España.

Villarroya, A. (2002). A look at school choice in Spain. Mediterranean Journal of Educational Studies, 7 (2), 21-36. https://bit.ly/2ktuPkP

\section{Para citar este artículo}

Bernal Agudo, J.L., y Vera Báez, C. (2019). La elección de centro como mecanismo de segregación social. Revista Fuentes, 21(2), 189-200. [Fecha de consulta: dd/mm/aa]. doi: 10.12795/revistafuentes.2019.v21.i2.04 